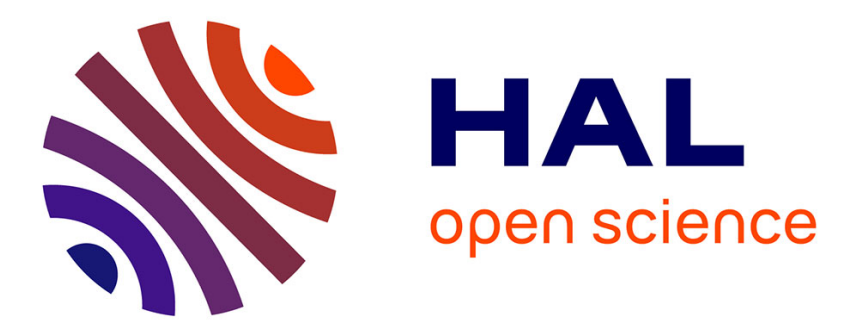

\title{
Principles and applications of a controllable electromagnetic band gap material to a conformable spherical radome
}

S. Haché, S.N. Burokur, A. de Lustrac, F. Gadot, P. Cailleu, G.-P. Piau

\section{- To cite this version:}

S. Haché, S.N. Burokur, A. de Lustrac, F. Gadot, P. Cailleu, et al.. Principles and applications of a controllable electromagnetic band gap material to a conformable spherical radome. European Physical Journal: Applied Physics, 2009, 46 (3), pp.1-7. 10.1051/epjap/2009040 . hal-00480163

\section{HAL Id: hal-00480163 https://hal.science/hal-00480163}

Submitted on 3 May 2010

HAL is a multi-disciplinary open access archive for the deposit and dissemination of scientific research documents, whether they are published or not. The documents may come from teaching and research institutions in France or abroad, or from public or private research centers.
L'archive ouverte pluridisciplinaire $\mathbf{H A L}$, est destinée au dépôt et à la diffusion de documents scientifiques de niveau recherche, publiés ou non, émanant des établissements d'enseignement et de recherche français ou étrangers, des laboratoires publics ou privés. 


\title{
Principles and Applications of a Controllable Electromagnetic Band Gap Material to a Conformable Spherical Radome.
}

\author{
S. Haché ${ }^{1}$, S. N. Burokur ${ }^{1}$, A. de Lustrac ${ }^{1}$, F. Gadot ${ }^{1}$, P. Cailleu ${ }^{2}$, G.-P. Piau ${ }^{2}$. \\ ${ }^{1}$ IEF, Paris Sud University, 91405 Orsay, France. \\ ${ }^{2}$ EADS Research Center, 92000 Suresnes, France
}

\begin{abstract}
This paper presents the principle of two types of conformable and controllable spherical radome based on Electromagnetic Band Gap (EBG) materials operating at around $10 \mathrm{GHz}$. The EBG structure is composed of a grid of metallic wires conformed on a hollow spherical object. Two switching control configurations are considered: 1) between an EBG structure made of electrically continuous wires and another one made of discontinuous wires, and 2) between two EBG structures made of discontinuous wires where each has a different period of discontinuities. Both switching configurations are simulated and experimentally characterized on passive prototypes. An excellent agreement is observed between simulations and measurements. The radiation patterns of two types of antennas, a horn antenna and a meteorological antenna, are also measured in the presence of the radome.
\end{abstract}

PACS: 41.20,Jb, 42.70.Qs, 84.40.Ba.

\section{Corresponding author:}

A. de Lustrac,

andre.de-lustrac@u-psud.fr,

Tel. 33169157854 .

Address : Institut d'Électronique Fondamentale, UMR 8622, Paris-Sud University, Bat 220, 91405 Orsay Cedex, France 
S. Haché, principles and applications of a CEBG to a spherical radome.

\section{Introduction}

Controllable Electromagnetic Band Gap (EBG) structures can find a variety of applications to antennas, RF screens, signature control and filters ... [1,2]. Several studies have been made on electronically active planar EBG structures in the microwave domain where their applications to electromagnetic windows and antennas have been demonstrated $[3,4]$. The main advantage of these structures resides in the large easiness of continuous directional control of transmission and of frequency adjustment, which depends on the structure's periodic parameters.

In this paper we use a controllable EBG to design an electronically controllable radome working around $10 \mathrm{GHz}$. In the open literature, Frequency Selective Surfaces (FSS) have been proposed to design radomes [5-11]. Patents covering the use of active FSS to design controllable radomes can also be found $[12,13]$. However to the best of our knowledge it is the first time that a controllable radome is made of a conformed EBG material. The application of controllable EBG material to a radome implies that the classical properties of a radome will be satisfied, i.e. a transmission close to unity. Then we have optimized the radome structure to meet this criterion.

The EBG structures considered in this paper are composed of periodically organized copper strips on a conformal spherical shape. The final aim of this study is to achieve an electronic commutation of the transmission from an allowed state to a forbidden one at around $10 \mathrm{GHz}$. The structure must then consist of discontinuous parallel copper strips incorporating fast switching elements such as PIN diodes or photoconductors, necessary to obtain the commutation of the metallic wires from a discontinuous electrical state to a continuous one. In this present work, we consider the spherical radome only in a passive mode. This study on passive structures is the starting point to demonstrate the principle of the commutation before going towards electronically active structures. EBG structures are characterized by an alternation of forbidden frequency and allowed frequency bands depending on the geometrical and electromagnetic parameters of the structure, and also on the polarization of the incident wave. If the EBG structure is made of continuous metallic wires parallel to the electrical field of the incident wave, the first band at low frequencies is a forbidden band. In a pioneering work, J. B. Pendry has studied metallic electromagnetic band gap materials and has shown that a continuous metallic lattice may be considered like a diluted metal with a first forbidden frequency band from $0 \mathrm{~Hz}$ to a plasma frequency $\omega_{\mathrm{p}}$ [14]. This plasma frequency depends on the geometrical parameters of the metallic lattice and on the used metal:

$$
\omega_{p}^{2}=\frac{n e^{2} \pi r^{2}}{\varepsilon_{0} a^{2} m_{\text {eff }}} \quad \text { with } \quad m_{\text {eff }}=\ln \left(\frac{a}{r}\right) \mu_{0} e^{2} r^{2} n / 2
$$

where $a$ is the period of the lattice, $r$ is the radius of the wire, $n$ is the electron density and $m_{\mathrm{eff}}$ the effective electron mass.

When the continuous metallic wires are replaced by discontinuous ones with discontinuities periodically inserted along the wires with a period $p$, an allowed band is observed at low frequencies. The bandwidth of this allowed band depends on the capacitance and the period of the discontinuities [15]. If we can control the switching of the metallic wires from a discontinuous electrical state to a continuous one, then we can obtain the switching of the structure from a transmitting state to a reflecting one. This is the first control configuration investigated in this paper (Fig.1a). However it is possible to use another mode. If we can control not only the electrical state of all the discontinuities, but also the periodicity of these 
discontinuities, we can then control the switching from a set of frequency bands, alternating forbidden and allowed bands, to a different set. If these periodicities are well chosen, an allowed band in the first set may be replaced by a forbidden band in the second set (Fig.1b). This constitutes the second control configuration under study. We can see in Fig. 1 that at the frequency $f_{0}$ both control modes allow the switching from a transmitting state of the EBG material to a reflecting one. Several conformed prototypes have been simulated, fabricated and characterized to illustrate both control modes on a real scale spherical radome. The results obtained from both configurations show that the switching of the transmission is observed in both cases in simulations and measurements with a very good agreement. The first configuration is more suitable to the use of electronic switching components biased by DC current whereas the second one corresponds much better to the use of photoconductive components.

In this study we present calculated and measured results obtained on only passive prototypes. These prototypes have been fabricated and characterized to demonstrate the feasibility of the electrical state switching concept. To further validate this concept, the radome has been tested with a meteorological radar antenna actually used in airplanes.

\section{Design and simulation of the EBG structures}

The radome considered here is a conformable one on a spherical shape. This shape corresponds to the vertex of the real radome of an ATR 42 airplane, with a maximum radius $R$ $=16.5 \mathrm{~cm}$. The studied EBG structures are all composed of a one-dimensional grid of parallel copper strips of width $w=1 \mathrm{~mm}$ (Fig. 2a) regularly disposed on the spherical shape with a projection period $a=1 \mathrm{~cm}$ on the horizontal plane (xOy plane). This structure has two symmetry planes $(\mathrm{xOz}$ and $\mathrm{yOz})$ that allow simplifying its simulation. The calculation is performed using Flomerics' Microstripes commercial software based on the Transmission Line Matrix method [16]. The software allows reducing the calculation time by simulating the wires using a wire model. The horn antenna used in the experimental characterizations of the radome is also included in the calculation to increase its accuracy. This type of excitation acts as a Transverse Electromagnetic (TEM) plane wave propagating towards the EBG structure in the $+z$ direction with its electric field $\mathbf{E}$ oriented along the $y$-axis and its magnetic field $\mathbf{H}$ oriented along the $x$-axis. The two switching control configurations are tested using structures with either continuous wires or discontinuous wires:

\subsection{Configuration 1: commutation between continuous and discontinuous wires}

The first spherical EBG structure is composed of continuous metallic wires of width $w=1$ $\mathrm{mm}$. The continuous wires are supposed to present an allowed band at $10 \mathrm{GHz}$. For the second structure composed of discontinuous wires, the discontinuities are placed along each row of wires in such a manner that their projections on the $y$-axis are equally spaced with a period $p$. The width of the discontinuities in all the rows is $0.1 \mathrm{~mm}$. For the simulation, the $\mathbf{E}-$ field is polarised parallel to the wires. Here, we have tested only this polarization, but it is also possible to design a structure operating for two perpendicular linear polarizations with the use of crossed grids at $90^{\circ}$. The wires are considered to be perfect conductors in the various simulations. The EBG structures are designed so as to obtain a switching between an allowed and a forbidden transmission band at around $10 \mathrm{GHz}$. In this first configuration, a commutation between the allowed band of the continuous wire strips structure and the forbidden band of discontinuous wire strips structure is desired. The position of the forbidden band depends on the discontinuities' periodicity and on the bend radius of the structure. 
On the sphere, each row of wires has a different bend radius. Since the transmission level through the EBG structure depends on this bend radius, a different discontinuities' periodicity $p_{1}$ must be applied to each row of wires in order to obtain a forbidden band at $10 \mathrm{GHz}$. The period $p_{1}$ for each row is determined separately from a cylindrical periodical structure, so as to obtain a commutation at the desired frequency. The cylindrical EBG structure has been previously presented and investigated in [17]. The different parameters of the structure for a forbidden band at around $10 \mathrm{GHz}$ are as follows: $p_{1}=11 \mathrm{~mm}$ (discontinuities' periodicity) for the first six rows of wires, near of the summit of the radome, $p_{1}=10 \mathrm{~mm}$ for the seventh $\left(7^{\text {th }}\right)$ to ninth $\left(9^{\text {th }}\right)$ rows and $p_{1}=9 \mathrm{~mm}$ for the last row $\left(10^{\text {th }}\right)$. This set of wires will be referred to as having a discontinuities' periodicity $p_{1}$ in the rest of the text.

Fig. $2 \mathrm{~b}$ presents the transmission responses calculated for each row of wires separately on a cylindrical structure having a different specified radius. These rows of wires will then be applied on the spherical radome of variable radius. The responses show a very low transmission level for each row at $10 \mathrm{GHz}( \pm 10 \%)$, indicating that the different optimized periods can be applied to the discontinuities in order to have a forbidden band with the spherical radome.

\subsection{Configuration 2: commutation between two structures of discontinuous wires}

In the second configuration a commutation between the allowed band of a structure composed of discontinuous wires with a projection period $p_{2}$ and the forbidden band of the structure composed of discontinuous wires with the projection period $p_{1}$ previously used in configuration 1. For this second structure composed of discontinuous wires, the same study to optimize the different values of $p_{2}$ has been done so to obtain an allowed band at $10 \mathrm{GHz}$. For the six first rows, the discontinuities periodicity is $p_{2}=5.5 \mathrm{~mm}$, for the $7^{\text {th }}$ to $9^{\text {th }}$ rows $p_{2}=5$ $\mathrm{mm}$, and for the last row $p_{2}=4.5 \mathrm{~mm}$. This set of wires will be referred to as having a discontinuities' periodicity $p_{2}$ in the rest of the text. The simulated transmission responses are presented in Fig. 2c. We can observe that the transmission level is high and close to $0 \mathrm{~dB}$ for each row of wires, indicating an allowed band at $10 \mathrm{GHz}$. These rows of wires will then be applied on the spherical radome to exhibit an allowed band.

The transmissions through the three EBG-based radomes normalized to that of the horn antenna are presented in Fig. 2d. For both configurations, a commutation of the transmitted signal can be observed at $10 \mathrm{GHz}$. This commutation reaches a level of more than $28 \mathrm{~dB}$. In the first configuration this switching occurs between the EBG structure made of continuous wires (solid grey curve) and the structure made of discontinuous wires with periods $p_{1}$ (dashed black curve). In the second configuration, the commutation is observed between the EBG structure made of discontinuous wires with periods $p_{1}$ and an EBG structure made of discontinuous wires with periods $p_{2}$ (solid light grey curve). The main difference between these two commutation configurations is the transmission level when the radome is transparent to the horn antenna. In the first configuration, this level is weaker $(-1.6 \mathrm{~dB}$ at 10 $\mathrm{GHz}$ ) for the EBG structure made of continuous wires than for the second one made of discontinuous wires with periods $p_{2}(-0.6 \mathrm{~dB})$.

\section{Measurements}

\subsection{Gain versus frequency}

Three different passive prototypes of the spherical EBG-based radome are fabricated according to the parameters mentioned above for the two switching control configurations. The first prototype is composed of continuous wire strips and the second one is composed of discontinuous wires with the periods of discontinuities $p_{1}$. Finally, the last prototype has the 
periods of discontinuities $p_{2}$ for the rows of wires. The wires made of copper are printed on a flexible support and are disposed on the hollow conformable spherical object made of low loss foam having a relative permittivity close to 1 and a thickness $h=17 \mathrm{~mm}$. The maximum radius of the sphere is $16.5 \mathrm{~cm}$. The widths of the wires and of the discontinuities are respectively $1 \mathrm{~mm}$ and $0.1 \mathrm{~mm}$. A first set of transmission measurements are done using a horn antenna working in the $\mathrm{X}$ band $[8.2 \mathrm{GHz}-12.4 \mathrm{GHz}]$ and having a angular beam width of $30^{\circ}$ at $-3 \mathrm{~dB}$. It is placed at the centre inside the hollow foam sphere at a distance of $20 \mathrm{~cm}$ of the EBG structure. A picture of the EBG-based spherical radome with the emitting horn antenna inside is shown in Fig. 3a. A receiving horn antenna is placed at a distance of $8 \mathrm{~m}$ from the radome. The measurements are done in the EADS Innovation Works' anechoic chamber at Suresnes (France) as illustrated by the experimental setup shown in Fig. 3b. The gains of the emitting horn antenna alone and in the presence of the three different EBG-based radomes are measured versus frequency and the results are presented in Fig. 4a.

The black curve represents the gain of the horn antenna alone in free space (without the use of the radome). For both configurations, a switching occurs at about $9.3 \mathrm{GHz}$. Compared to the simulation results, the measured commutation is shifted towards lower frequencies of about $0.7 \mathrm{GHz}$. This frequency shift between the simulated and the measured responses can be explained by the fabrication accuracy of the structures. The grey curve represents the gain response for the EBG structure with continuous wires. We can note that there is an average loss of $1.5 \mathrm{~dB}$ compared to the gain of the antenna alone, as it has been observed in simulations. In the first configuration, the switching between the EBG structures made of continuous wires and discontinuous wires with periods $p_{1}$ reaches $24.5 \mathrm{~dB}$, slightly weaker than the simulated one. The light grey curve represents the gain response for the radome made of discontinuous wires with periods $p_{2}$. Compared to the gain of the horn antenna alone, the transmission is quite lossless at $9.3 \mathrm{GHz}$. In the second configuration, the switching between the two EBG structures composed of discontinuous wires reaches a level of more than $26 \mathrm{~dB}$.

A second set of measurements are done using as emitter a meteorological radar antenna array working at a centre frequency of $9.3 \mathrm{GHz}$ with a bandwidth of about $10 \%$ [8.8 GHz -9.8 $\mathrm{GHz}$ ] and having a angular beam width of $12^{\circ}$ at $-3 \mathrm{~dB}$. This antenna is composed of an array of 256 elementary slot antennas. This type of antenna is used in the modern aircraft for weather forecasts and corresponds much better to the antenna associated really with this radome. In this case, the switching reaches a level of about $16 \mathrm{~dB}$ as shown in Fig. 4(b). Compared to the gain of the meteorological antenna alone, there is an average loss of $2 \mathrm{~dB}$ when the EBG-based radome is transparent.

\subsection{Radiation patterns}

The E-plane radiation patterns are also measured at the frequency corresponding to the commutation ( $f=9.3 \mathrm{GHz}$ ) for the horn antenna alone and in presence of the three EBGbased radomes. The diagrams are shown in Fig. 5a where the same color code of Fig. 4 holds. In these measurements, the radome is rotated from $-120^{\circ}$ to $120^{\circ}$ with respect to the position of the horn antenna which remains fixed. From Fig. 5a, we can observe that the primary lobes of the continuous wires and the discontinuous wires with period $p_{2}$ have the same aperture angle at $-3 \mathrm{~dB}$ as that of the horn antenna alone. However the secondary lobes reaching a level of $-12 \mathrm{~dB}$ are higher for the EBG structure with period $p_{2}$. This may be due to the large number of discontinuities in this structure.

As for the horn antenna, measurements are done with the three EBG structures associated with the meteorological antenna array in order to show the commutation of the transmitted signal. The radiation patterns of the antenna alone and in presence of the EBG-based radomes 
S. Haché, principles and applications of a CEBG to a spherical radome.

at $9.3 \mathrm{GHz}$ are presented in Fig. 5b. Concerning the first commutation configuration, i.e. between the continuous wires and the discontinuous ones with periods $p_{1}$, we can observe a maximum level of commutation in transmission at $9.3 \mathrm{GHz}$ of about $16 \mathrm{~dB}$. For the second configuration between the EBG structure composed of discontinuous wires with periods $p_{2}$ and the one with $p_{1}$, the commutation level is quite identical. We can also observe an increase in the level of the secondary lobes in the presence of the radome. But the level of these lobes lies under $-20 \mathrm{~dB}$, which is better than those observed in the measurements using the horn antenna as emitter.

\section{Conclusion}

This paper presents the principle of two types of conformable and controllable spherical radomes based on an Electromagnetic Band Gap (EBG) material operating at around $9.3 \mathrm{GHz}$. The EBG structures are composed of a grid of metallic wires conformed on a hollow spherical object. Two configurations corresponding to two different operating modes have been tested: 1) an EBG structure made of electrically continuous wires and a structure made of discontinuous wires with periods $p_{1}, 2$ ) two EBG structures made of discontinuous wires with different periods of discontinuity, $p_{1}$ and $p_{2}$. Calculations and measurements performed on passive prototypes are in good agreement. A switching of the transmission is observed in simulations at $10 \mathrm{GHz}$ and in measurements at $9.3 \mathrm{GHz}$. These EBG-based radomes have been tested with a horn antenna and a meteorological antenna array composed of 256 elementary slot antennas. The radiation patterns of both configurations of radomes have been measured and presented. A switching of about $26 \mathrm{~dB}$ is observed for the horn antenna, with an increase of the secondary lobes at a level of $-12 \mathrm{~dB}$. For the second antenna, which corresponds better to the real antenna used with this type of radome, we obtain a switching of $16 \mathrm{~dB}$, with secondary lobes lying under $-20 \mathrm{~dB}$.

Acknowledgement: The authors would like to thank the EADS Foundation and DGA for its financial support. 
S. Haché, principles and applications of a CEBG to a spherical radome.

\section{References}

[1] M. Hook, J. Vardaxoglou, K. Ward, Proc. 27th ESA Antenna Technology Workshop Innovative Periodic Antennas, Santiago de Compostela, Spain, Mar. 2004, pp. 273-283.

[2] S. Maci, M. Caiazzo, A. Cucini, M. Casaletti, IEEE Trans. Antennas Propag. 53, no.1, pp. 70-81 (2005).

[3] A. de Lustrac, F. Gadot, S. Cabaret, J.-M. Lourtioz, T. Brillat, A. Priou, E. Akmansoy, Appl. Phys. Lett., vol. 75, no. 11, 1625, Sept. 1999.

[4] A. de Lustrac, F. Gadot, E. Akmansoy, T. Brillat, Appl. Phys. Lett., vol. 78, no. 26, 4196, June 2001.

[5] B. A. Munk, "Frequency selective surfaces, Theory and design", Wiley-Interscience publication, p.259, 2000.

[6] T.K. Chang, R.J. Langley, and E. Parker, IEEE Microw. Guid. Wave Lett., vol.3, no.10, pp.387-388, Oct. 1993.

[7] B. Philips, E.A. Parker, and R.J. Langley, Electron. Lett., vol.31, no.1, pp.1-2, Jan. 1995.

[8] T.K. Chang, R.J. Langley, and E.A. Parker, IEE Proc. Microwaves Antennas and Propagation, vol.143, no.1, pp.62-66, Feb. 1996.

[9] J.C. Vardaxoglou, Electron. Lett., vol.32, no.25, pp.2345-2346, Dec. 1996.

[10] B.M. Cahill and E.A. Parker, Electron. Lett., vol.37, no.4, pp.244-245, Feb. 2001.

[11] Ghaffer I. Kiani, Kenneth L. Ford, Karu P. Esselle, Andrew R. Weily, C. Panagamuwa, John C. Batchelor, Microwave and Optical Technology Letters, Volume 50, Issue 8, August 2008, Pages: 2149-2151

[12] Whelan, David A., Fraschilla, John Pierce, Brian M. , "Ferro-electric frequency selective surface radome", United States Patent 5600325,1997.

[13] Arceneaux, Walter S., Akins, Rickey D. May, Walter B., "Absorptive/transmissive radome", United States Patent 5400043, 1995.

[14] J.B. Pendry, A. Holden, W. Stewart, and I. Youngs, Phys. Rev. Lett., Vol. 76, 4773, June 1996.

[15] A. de Lustrac, T . Brillat , F . Gadot, E . Akmansoy, Optical and Quantum Electronics 34: 265-277, 2002.

[16] Microstripes, Microstripes Reference Manual Release 7.0, FLOMERICS Ltd., 2005.

[17] S. Haché, S. N. Burokur, F. Gadot, P. Cailleu, G.-P. Piau, A. de Lustrac, Proc. Loughborough Antennas and Propagation Conference (LAPC) 2007, Loughborough, UK, Apr. 2004, p.305. 
S. Haché, principles and applications of a CEBG to a spherical radome.

\section{Figures captions}

Fig. 1(a): First operating mode: switching between an EBG structure composed of continuous metallic wires (1:grey curve) and one made of discontinuous metallic wires with a period $p_{1}$ of discontinuities (2: black curve). (b) Second operating mode: switching between an EBG structure composed of discontinuous metallic wires with a period $p_{1}$ of discontinuities (1: black curve)and another one with a period $p_{2}$ of discontinuities (2: grey curve). At the frequency $f_{0}$ both operating modes allow the switching from a transmitting state of the EBG structure to a reflecting one.

Fig. 2(a): Schematic figure of the simulated structure with Microstripes. The inset shows half of the simulated radome in the $\mathrm{xOz}$ plane with the position of the different rows of wires. (b) Calculated transmission response for different row of wires with different periods $p_{1}$. (c) Calculated transmission response for different row of wires with different periods $p_{2}$. (d) Calculated frequency response for both configurations of wires. The radome is transmitting when the wires are continuous (grey curve) and when they are discontinuous with a discontinuity period $p_{2}$ (solid light grey curve). The radome is reflecting when the wires are discontinuous with a period $p_{1}$ (dark dashed curve). The commutation reaches more than 28 $\mathrm{dB}$ at $10 \mathrm{GHz}$ between the transmitting and the reflecting state. The transmission of the horn antenna alone is given by the black curve.

Fig. 3: (a) EBG structure under test in the anechoic chamber of EADS (Suresnes, France). (b) Schematic view of the anechoic chamber with the radome under test.

Fig. 4: (a) Measured transmission responses for the two commutation configurations with the horn antenna. (b) Measured transmission responses for the two commutation configurations with the meteorological antenna. The EBG radome is transmitting when the wires are continuous (solid grey curve) and when they are discontinuous with a discontinuity period $p_{2}$ (light grey curve). The radome is reflecting when the wires are discontinuous with a period $p_{1}$ (dashed black curve). The solid black curve illustrates the gain of the horn antenna alone.

Fig. 5: (a) Radiation patterns for the three EBG radomes and the horn antenna alone at 9.3 GHz. (b) Radiation patterns for the three EBG radomes and the meteorological antenna alone at $9.3 \mathrm{GHz}$. The color code is the same as in Fig. 4. 
S. Haché, principles and applications of a CEBG to a spherical radome.

Figure 1
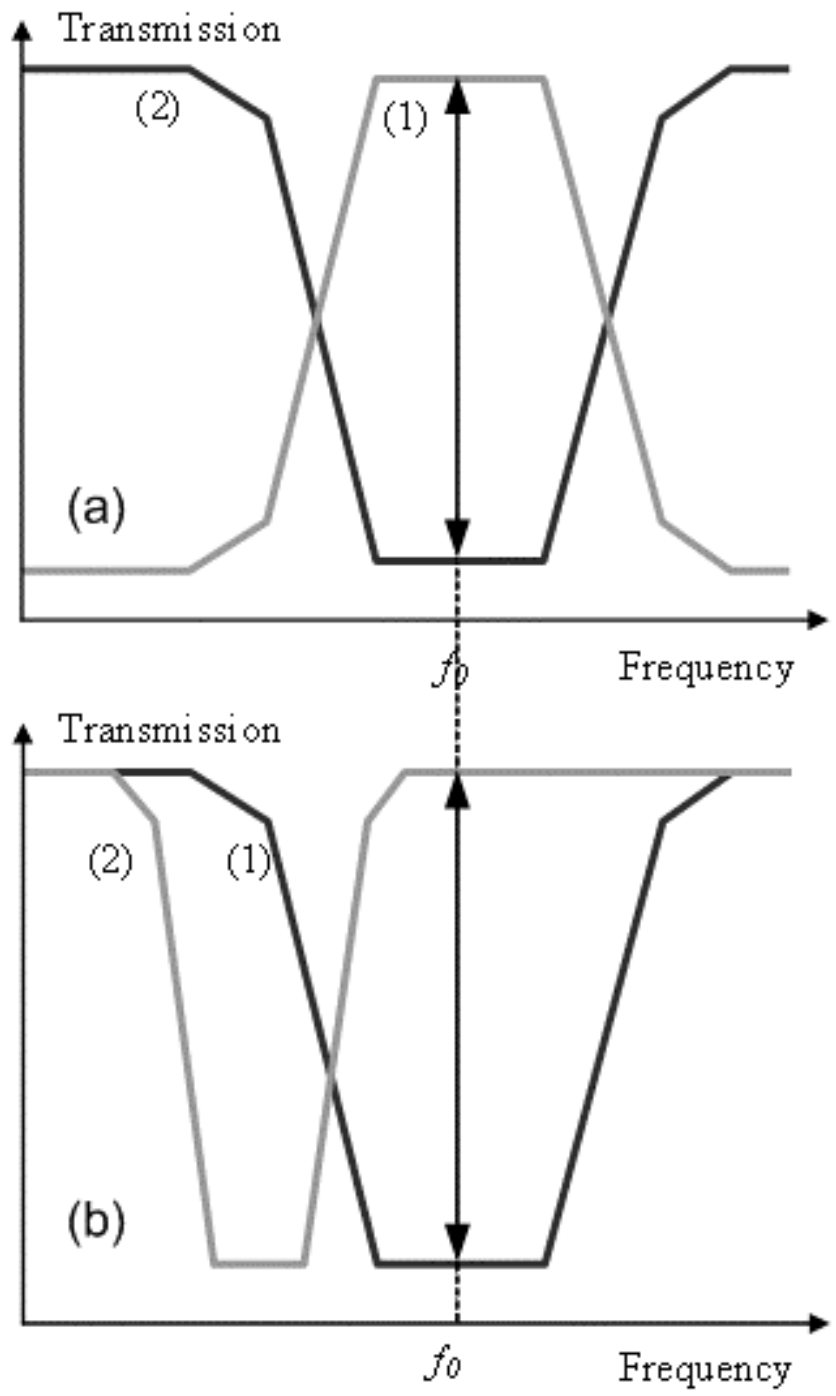
S. Haché, principles and applications of a CEBG to a spherical radome.

Figure 2a
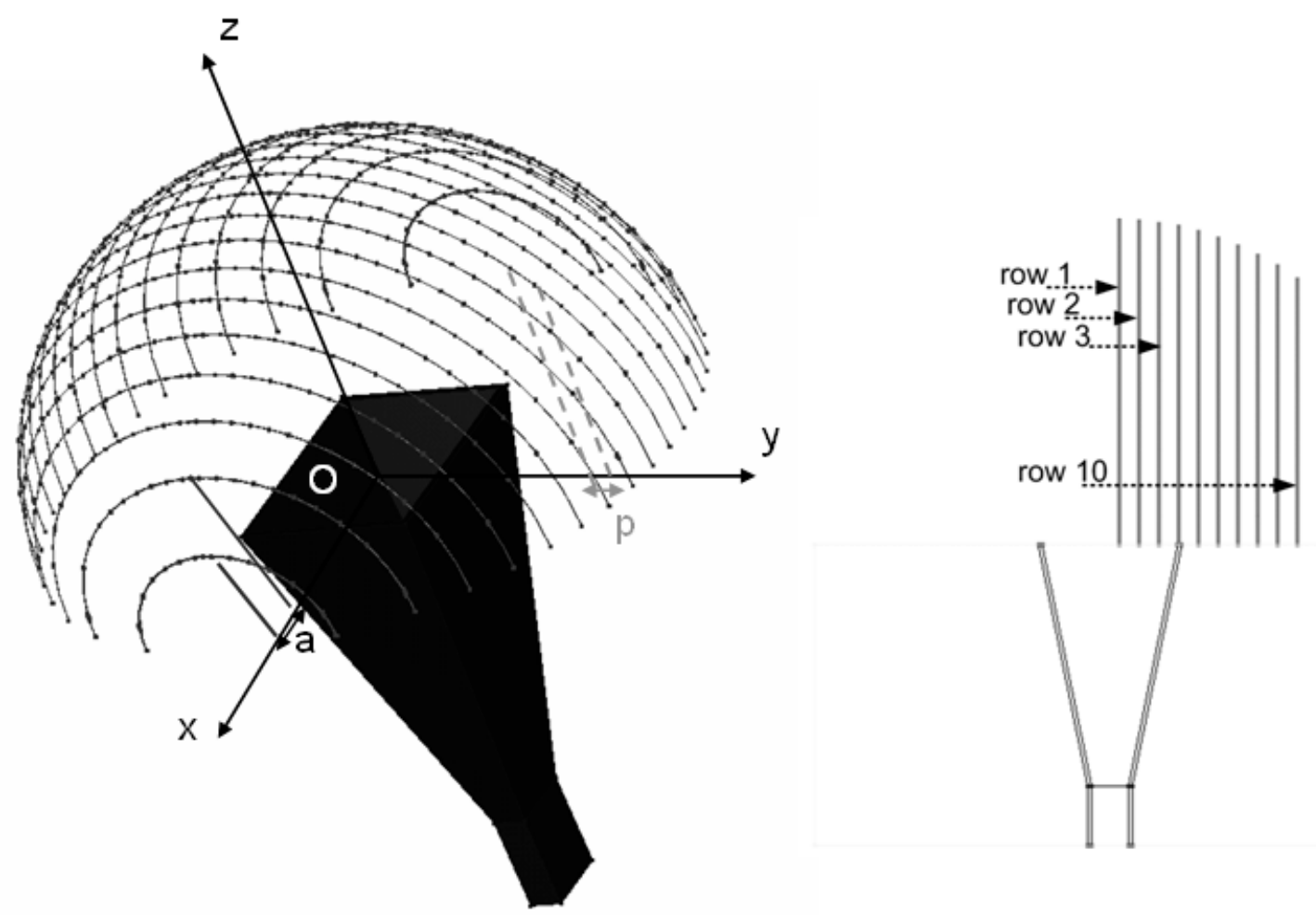
S. Haché, principles and applications of a CEBG to a spherical radome.

Figure $2 b$

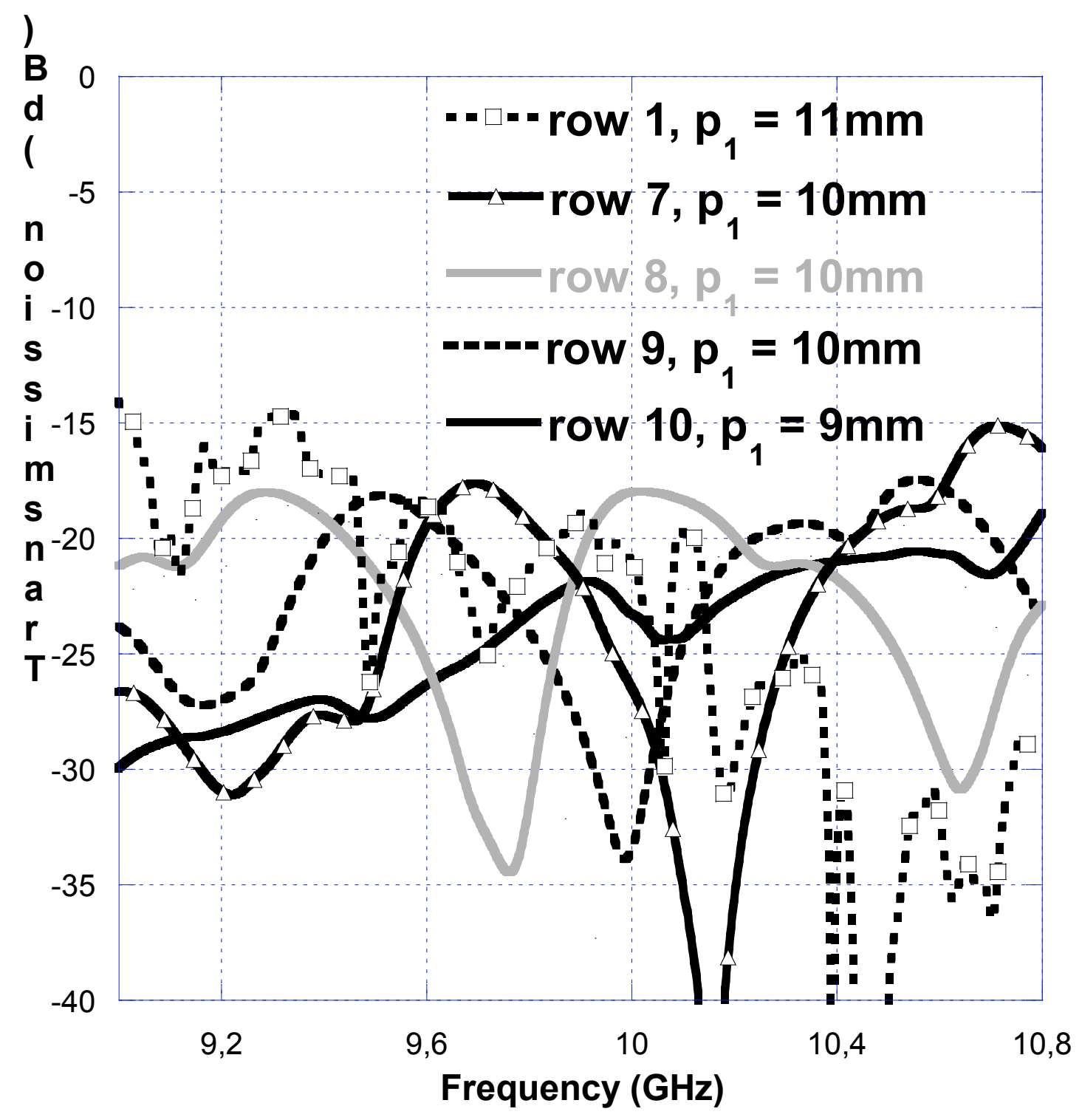


S. Haché, principles and applications of a CEBG to a spherical radome.

Figure 2c

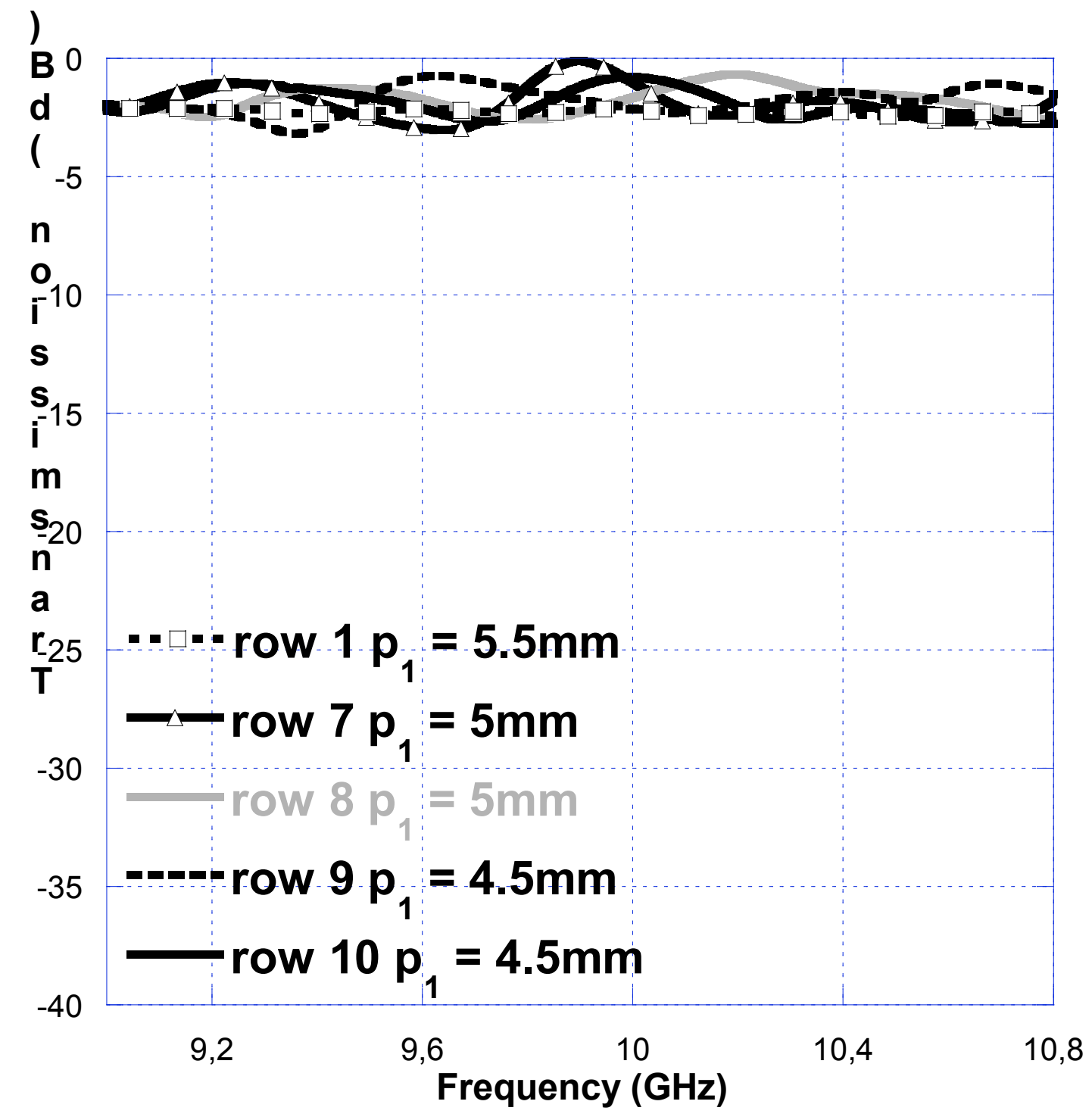


S. Haché, principles and applications of a CEBG to a spherical radome.

Figure 2d

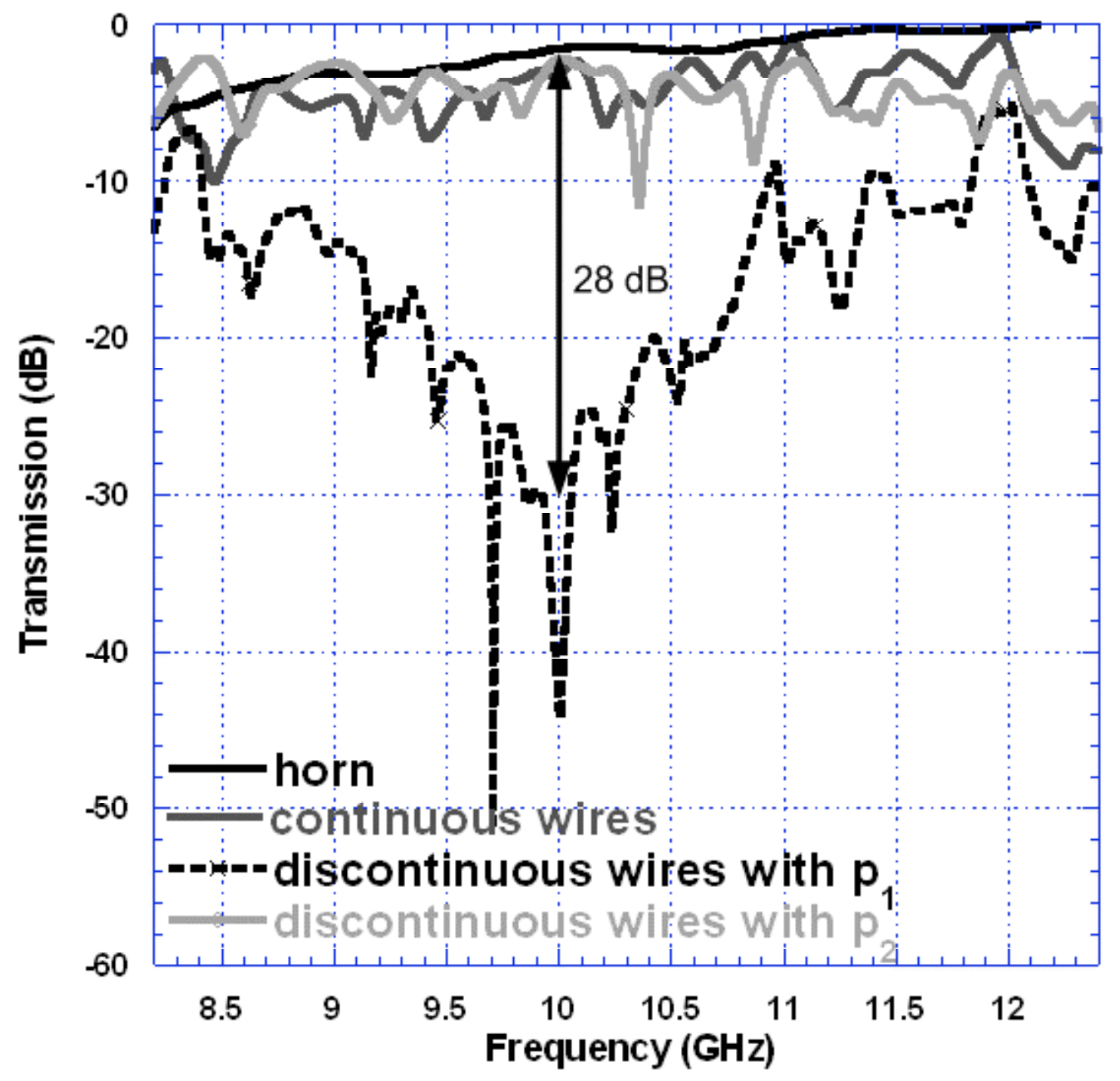


S. Haché, principles and applications of a CEBG to a spherical radome.

Figure $3 \mathrm{a}$

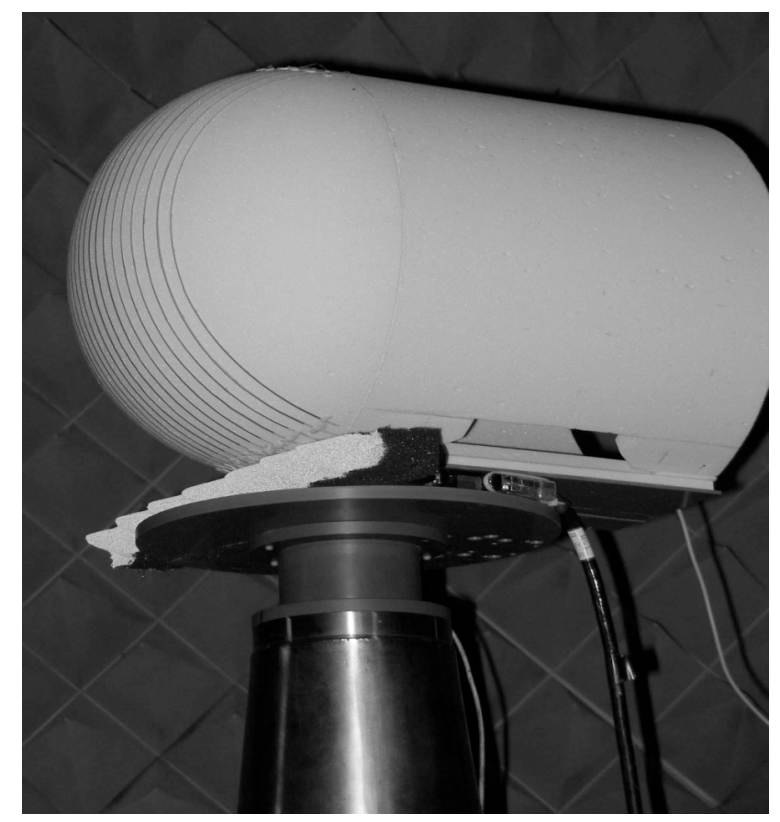

Figure $3 b$

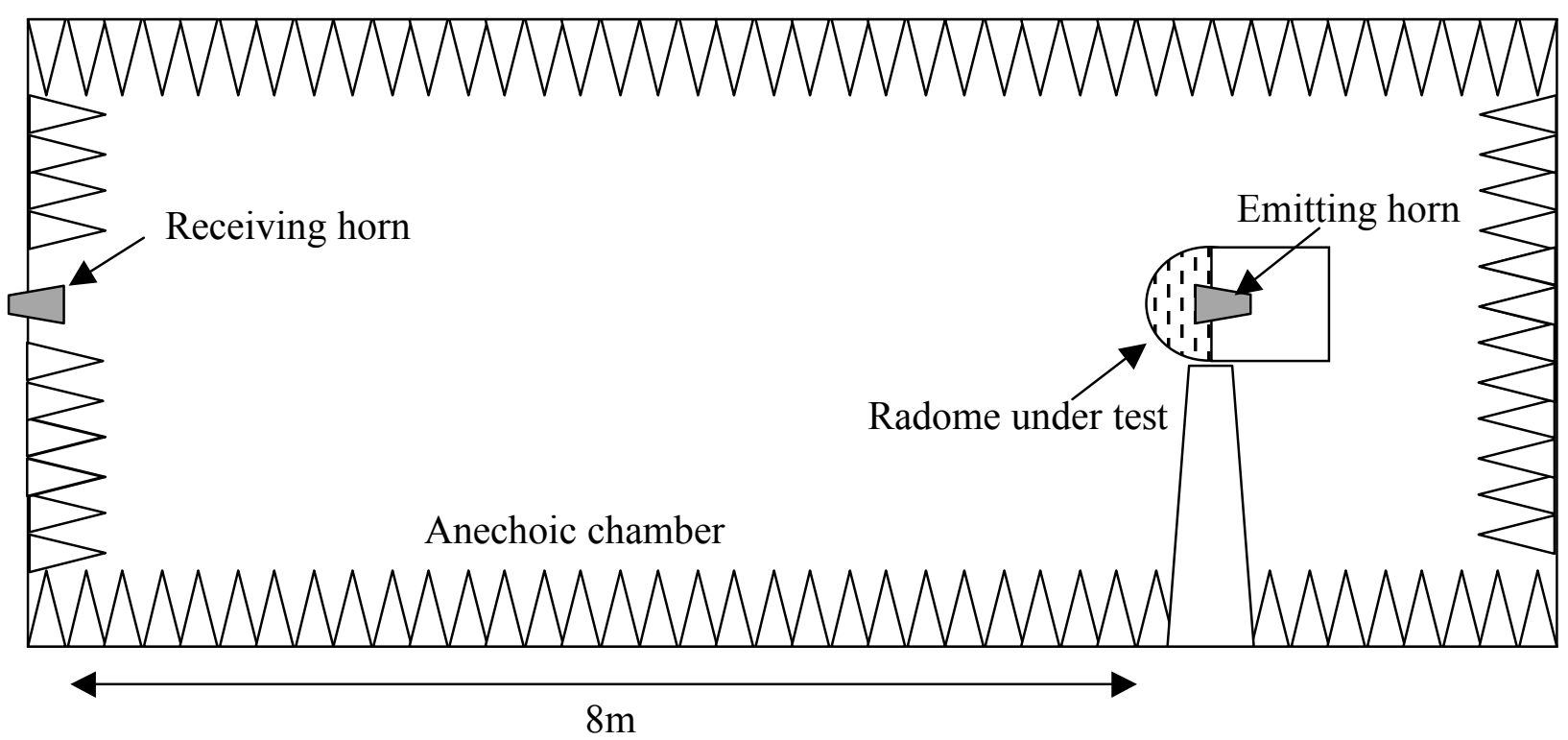


S. Haché, principles and applications of a CEBG to a spherical radome.

Figure $4 \mathrm{a}$

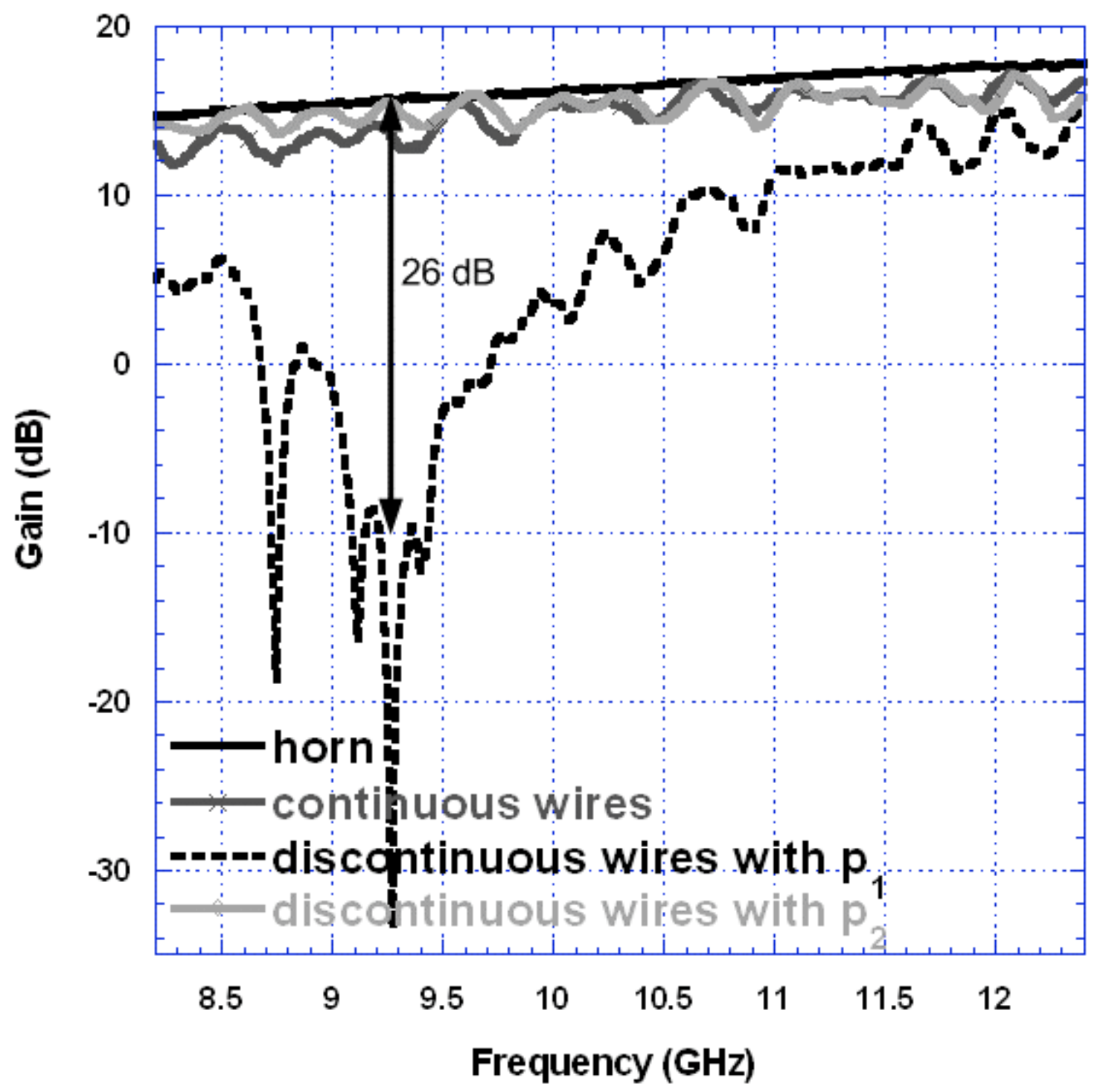


S. Haché, principles and applications of a CEBG to a spherical radome.

Figure4b

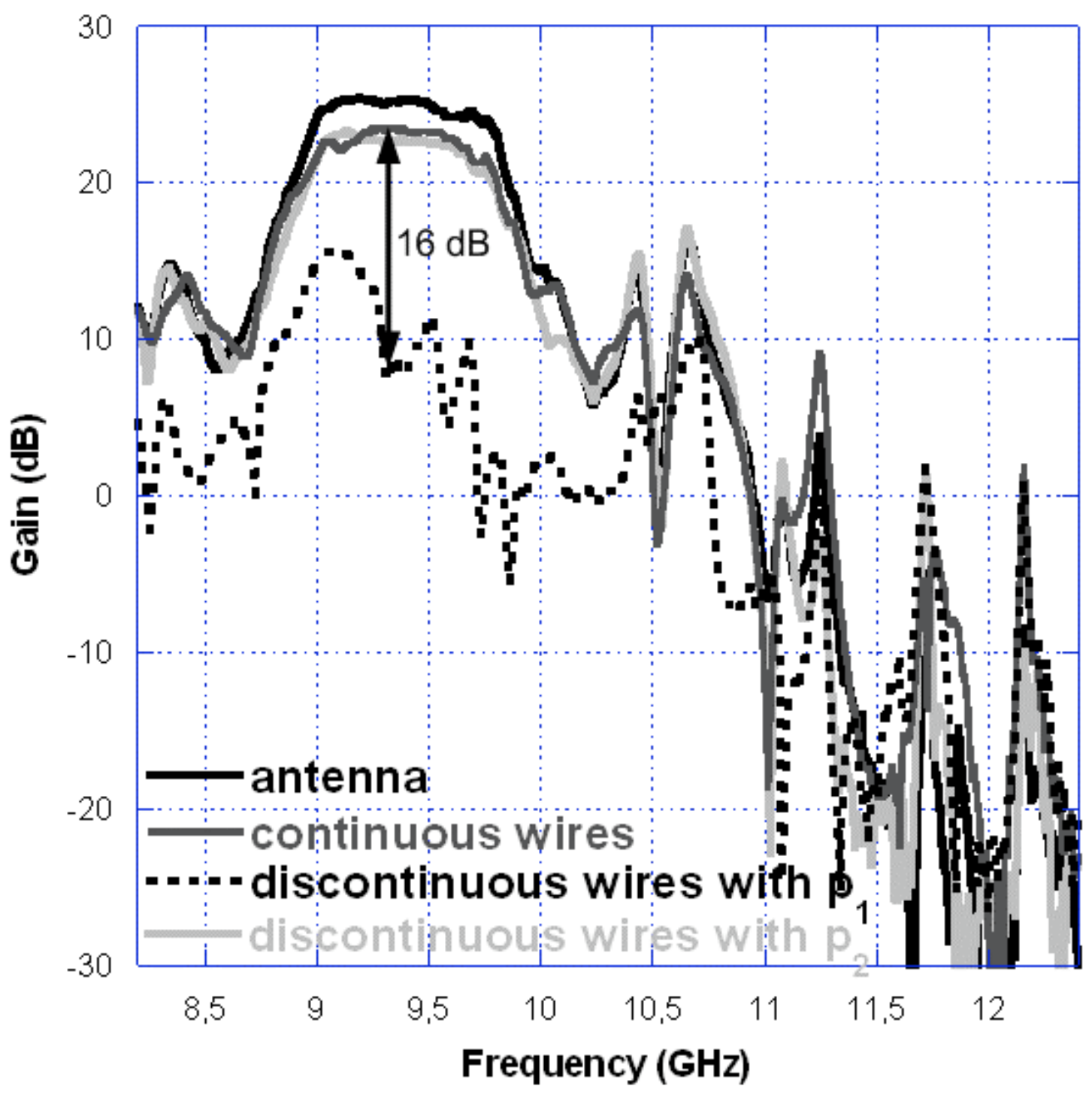


S. Haché, principles and applications of a CEBG to a spherical radome.

Figure 5a

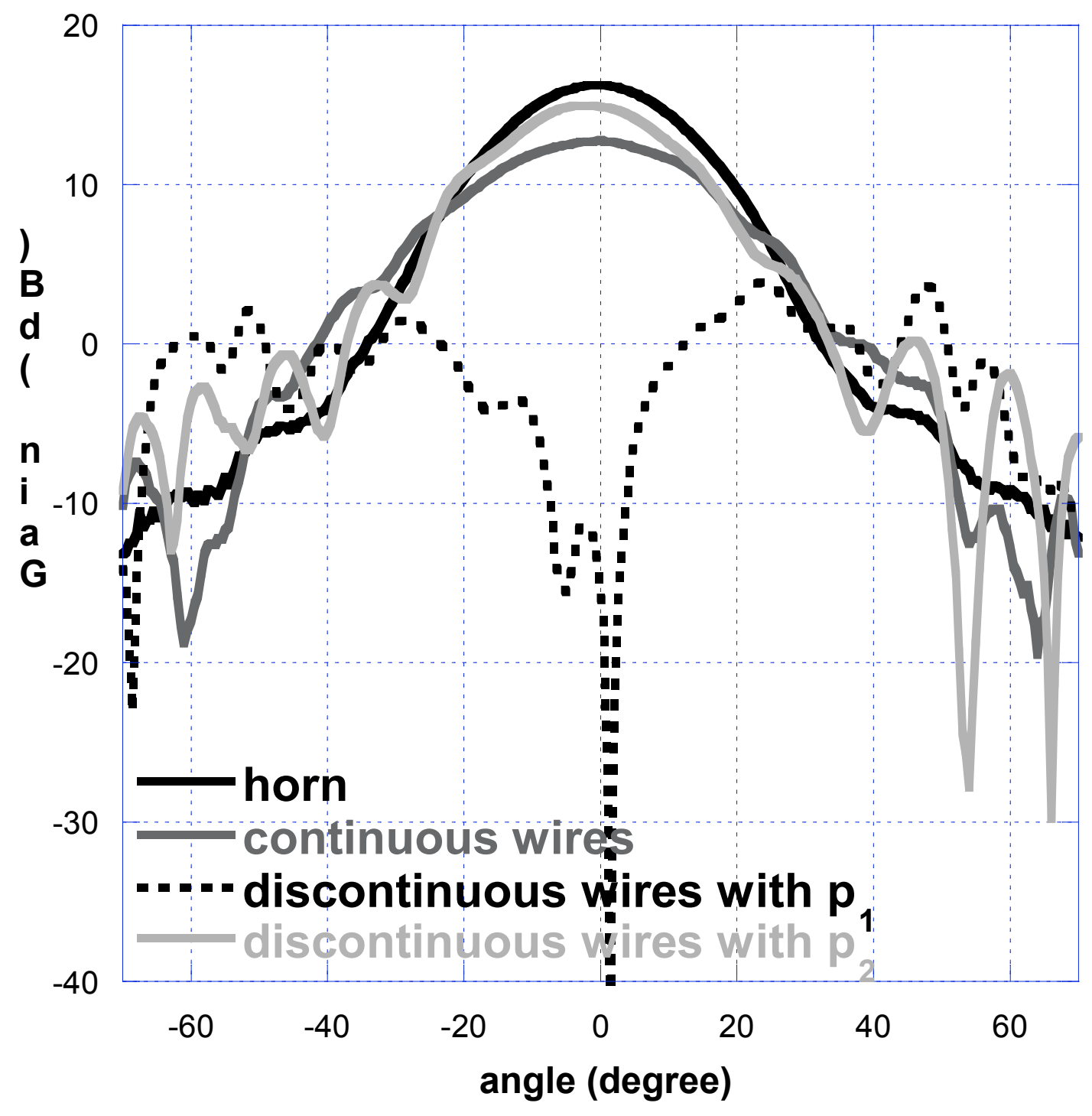


S. Haché, principles and applications of a CEBG to a spherical radome.

Figure $5 b$

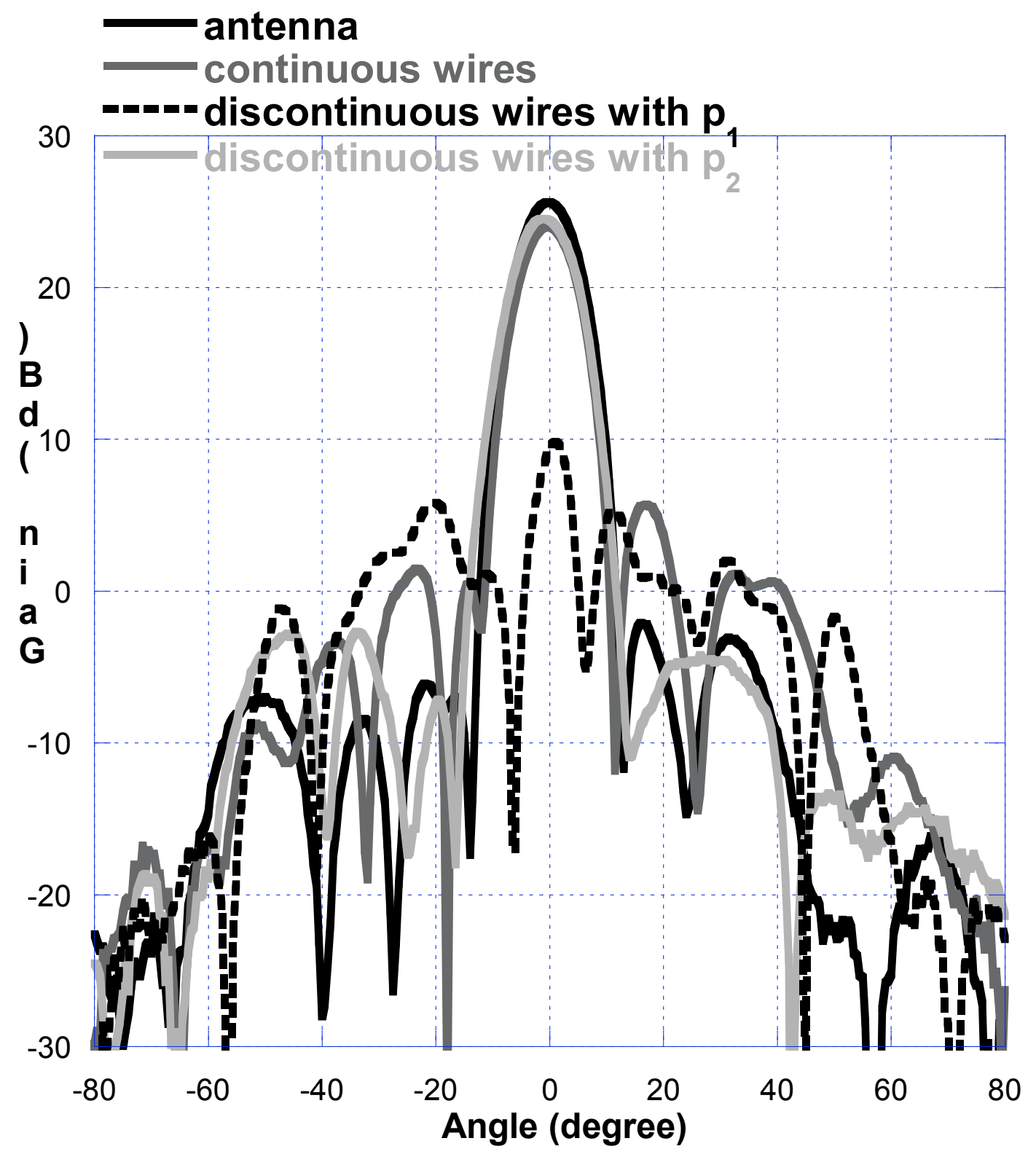

